\title{
Equivalence between Non-Markovian and Markovian Dynamics in Epidemic Spreading Processes
}

\author{
Michele Starnini, ${ }^{1,2, *}$ James P. Gleeson, ${ }^{3}$ and Marián Boguñã ${ }^{1,2, \dagger}$ \\ ${ }^{1}$ Departament de Física de la Matèria Condensada, Universitat de Barcelona, Martí i Franquès 1, E-08028 Barcelona, Spain \\ ${ }^{2}$ Universitat de Barcelona Institute of Complex Systems (UBICS), Universitat de Barcelona, 08028 Barcelona, Spain \\ ${ }^{3}$ MACSI, Department of Mathematics and Statistics, University of Limerick, Limerick, Ireland \\ (Received 10 January 2017; revised manuscript received 13 February 2017; published 24 March 2017)
}

\begin{abstract}
A general formalism is introduced to allow the steady state of non-Markovian processes on networks to be reduced to equivalent Markovian processes on the same substrates. The example of an epidemic spreading process is considered in detail, where all the non-Markovian aspects are shown to be captured within a single parameter, the effective infection rate. Remarkably, this result is independent of the topology of the underlying network, as demonstrated by numerical simulations on two-dimensional lattices and various types of random networks. Furthermore, an analytic approximation for the effective infection rate is introduced, which enables the calculation of the critical point and of the critical exponents for the non-Markovian dynamics.
\end{abstract}

DOI: 10.1103/PhysRevLett.118.128301

Modeling the stochastic dynamics that occur in many natural and technological systems has long depended on the Markovian assumption. In a Markov process, the probabilities of the occurrence of future events depend only on the present state of the system, being independent of the prior history. This memoryless property implies that such dynamics can be described by Poisson processes with fixed rates, which are characterized by an exponential distribution of the interevent time between consecutive events [1]. The mathematical tractability of Markov processes enables great simplifications in problem formulation, leading to important successes in the description of many dynamical processes unfolding on networks [2] and in other complex systems.

The dominance of the Markovian modeling framework has recently been challenged by the increasing availability of time-resolved data on different kinds of interactions, ranging from human activity patterns, including communication and mobility [3-5], to natural phenomena [6,7], biological processes [8], and biochemical reactions [9]. These empirical observations have revealed correlated sequences of events with heavy tailed interevent time distributions [10], which is a clear signature that the homogeneous temporal process description is inadequate and that non-Markovian (NM) dynamics lie at the core of such interactions.

Meanwhile, the interest in non-Markovian dynamical processes within the complex systems community has blossomed, from the points of view of both mathematical modeling [11-16] and numerical simulation [17,18]. Particular attention has been devoted to epidemic spreading on complex networks, representing the diffusion of information or disease in a population [19]. Recently, it has been shown that a non-Markovian infection dynamics dramatically alters the susceptible-infected-susceptible (SIS) spreading process [17,20,21]. Non-Markovian effects are now known to give qualitatively new behavior in information spreading, e.g., on social networks, as revealed by measurements of interevent times for email responses [22,23] and retweets on Twitter [24]. In the context of epidemiology, the non-Markovian assumption is particularly relevant, as empirical measurements of real diseases - smallpox, measles, and ebola—indicate that the distribution of infectious periods is far from being exponential [25-28].

In this Letter, we consider a SIS epidemic model controlled by a non-Markovian infection process and show that its steady state is equivalent to a Markovian one with an effective infection rate $\lambda_{\text {eff }}$, thus encoding all the nonMarkovian effects into a single parameter. Interestingly, such equivalence is independent of the underlying network topology. Our mathematical formalism demonstrates the existence of the effective rate $\lambda_{\text {eff }}$, allowing us to compute it by means of numerical simulations, and enables us to derive an approximate analytic expression $\lambda_{\text {app }}$, in very good agreement with $\lambda_{\text {eff }}$. Moreover, the approximate value $\lambda_{\text {app }}$ is expected to exactly converge to $\lambda_{\text {eff }}$ in the limit of low prevalence, thus close to the epidemic threshold. Therefore, the critical point and the set of critical exponents of the non-Markovian SIS dynamics, when expressed in terms of $\lambda_{\text {app }}$, are the same of those of the Markovian case, as we show by means of a finite size scaling analysis.

Let us consider an undirected and unweighted network topology defined by an adjacency matrix $a_{i j}$, with $i, j=$ $1, \ldots, N$, and a general, non-Markovian SIS dynamics running on top. In this model, nodes exist in either of two states, susceptible or infected. Infected nodes decay spontaneously to the susceptible state after a random time $t$ 
distributed as $\psi_{R}(t)$, that is, recovery from the illness does not confer any long lasting immunity, a characteristic present in some sexually transmitted diseases [29]. Susceptible nodes may become infected upon contact with infected neighbors. We assume that each infectious (or active) link, connecting an infected node with a susceptible one, hosts statistically independent stochastic infection processes, each one controlled by the same interevent distribution $\psi_{I}(t)$. In an active link isolated from the rest of the system, the susceptible node becomes infected after a random time $t$ has elapsed since the infection was initiated, with $t$ distributed as $\psi_{I}(t)$. If a susceptible node is connected to more than one infected neighbor, infection processes take place independently along each infectious link.

Distributions $\psi_{R}(t)$ and $\psi_{I}(t)$ allow us to evaluate the (time-dependent) hazard rates, defined as the probability per unit of time that, given that the event did not take place by a time $t$ since the process was initiated, it takes place in the time interval between $t$ and $t+d t$ [30]. The recovery and infection hazard rates are defined as $\delta(t)=$ $\psi_{R}(t) / \Psi_{R}(t)$ and $\lambda(t)=\psi_{I}(t) / \Psi_{I}(t)$, where $\Psi_{R}(t)$ and $\Psi_{I}(t)$ are the corresponding survival probabilities, that is, the probability that a given event takes a time longer than $t$. When temporal processes follow Poisson (Markovian) statistics, both distributions are exponential and the corresponding hazard rates are constants.

The SIS dynamics can be fully described by a set of binary stochastic processes $\left\{n_{i}(t)\right\}, i=1, \ldots, N$, defined as $n_{i}(t)=1$ if node $i$ is infected at time $t$ and 0 if it is susceptible. The exact stochastic evolution of these processes can be written as

$$
n_{i}(t+d t)=n_{i}(t) \xi_{i}(t, d t)+\left[1-n_{i}(t)\right] \eta_{i}(t, d t) .
$$

In this equation, the first term in the sum of the right-hand side is different from 0 only when node $i$ is infected and accounts for its recovery during the time interval $(t, t+d t)$. To achieve this, the stochastic process $\xi_{i}(t, d t)$ is defined to be equal to 0 with probability $d t \delta\left[t_{i}(t)\right]$ and one otherwise, where $t_{i}(t)$ is the time elapsed, at time $t$, since node $i$ became infected. Similarly, the second term in the sum of the right-hand side of Eq. (1) accounts for the infection of susceptible node $i$ by one of its infected neighbors during the time interval $(t, t+d t)$. The stochastic process $\eta_{i}(t, d t)$ is defined to be equal to 1 with probability $d t \sum_{j} a_{i j} n_{j}(t) \lambda\left[\tau_{j i}(t)\right]$ and 0 otherwise, where $\tau_{j i}(t)$ is the time elapsed since the infection process of node $j$ to node $i$ started. Note that we implicitly assume that each infected neighbor defines a statistically independent random process so that the total infection hazard rate is simply the sum of the infection hazard rates of each individual process. Note also that in this formulation $t_{i}(t)$ and $\tau_{j i}(t)$ are themselves stochastic processes.
The average of Eq. (1), first conditioned to the knowledge of the stochastic processes $\left\{n_{i}, t_{i}, \tau_{j i}\right\}$ at time $t$, and then over the unconditional values, allows us to write the following differential equation for the probability of node $i$ to be infected at time $t, \rho_{i}(t) \equiv\left\langle n_{i}(t)\right\rangle$,

$$
\begin{aligned}
\dot{\rho}_{i}(t)= & -\left\langle n_{i}(t) \delta\left[t_{i}(t)\right]\right\rangle \\
& +\sum_{j=1}^{N} a_{i j}\left\langle\left[1-n_{i}(t)\right] n_{j}(t) \lambda\left[\tau_{j i}(t)\right]\right\rangle .
\end{aligned}
$$

Note that with this definition, the prevalence of the disease at time $t$ is simply given by $\rho(t)=N^{-1} \sum_{i=1}^{N} \rho_{i}(t)$. The first term in Eq. (2) can be rewritten as (see Supplemental Material [31])

$$
\left\langle n_{i}(t) \delta\left(t_{i}(t)\right)\right\rangle=\rho_{i}(t)\left\langle\delta\left[t_{i}(t)\right] \mid n_{i}=1\right\rangle .
$$

In the limit $t \rightarrow \infty$, the only information we have about $t_{i}$, given that node $i$ is infected, is that the recovery time of node $i$ after infection is longer than $t_{i}$. This implies that the probability density of $t_{i}$ is given by $\Psi_{R}\left(t_{i}\right) /\left\langle t_{R}\right\rangle$, where $\left\langle t_{R}\right\rangle$ is the average recovery time [30]. By combining this result with the form of the recovery hazard rate, we can write

$\lim _{t \rightarrow \infty}\left\langle n_{i}(t) \delta\left(t_{i}(t)\right)\right\rangle=\rho_{i}^{s t} \int_{0}^{\infty} \frac{\Psi_{R}\left(t_{i}\right)}{\left\langle t_{R}\right\rangle} \delta\left(t_{i}\right) d t_{i}=\frac{\rho_{i}^{s t}}{\left\langle t_{R}\right\rangle}$,

where we have defined $\rho_{i}^{s t}=\lim _{t \rightarrow \infty} \rho_{i}(t)$. Similarly, the terms on the right-hand side of Eq. (2) can be written as

$$
\begin{aligned}
& \left\langle\left[1-n_{i}(t)\right] n_{j}(t) \lambda\left[\tau_{j i}(t)\right]\right\rangle \\
& \quad=\left\langle\left[1-n_{i}(t)\right] n_{j}(t)\right\rangle\left\langle\lambda\left[\tau_{j i}(t)\right] \mid n_{i}=0, n_{j}=1\right\rangle .
\end{aligned}
$$

From this equation, we observe that the evolution of the density $\rho_{i}(t)$ depends on the evolution of two-point correlation functions, $\rho_{i j}(t)=\left\langle n_{i}(t) n_{j}(t)\right\rangle$, that appear in the second term of Eq. (2). Using similar arguments to those used to derive Eq. (2), we can write an exact differential equation for the $n$-point correlation function $\rho_{i_{1} \cdots i_{n}}$ (see Supplemental Material [31]),

$\dot{\rho}_{i_{1} \cdots i_{n}}=\sum_{i \in \mathcal{I}}\left\langle\left(-\delta\left(t_{i}\right) n_{i}+\left(1-n_{i}\right) \sum_{j=1}^{N} a_{i j} n_{j} \lambda\left(\tau_{j i}\right)\right) \prod_{k \in \mathcal{I}_{i}} n_{k}\right\rangle$,

where we omit the dependence on $t$ for brevity and we define the sets of nodes $\mathcal{I} \equiv\left\{i_{1}, i_{2}, \cdots i_{n}\right\}$ and $\mathcal{I}_{i} \equiv \mathcal{I} \backslash i$. Eq. (6) can be written as

$\dot{\rho}_{i_{1} \cdots i_{n}}=-\rho_{i_{1} \cdots i_{n}} \sum_{i \in \mathcal{I}} \tilde{\delta}_{i}+\sum_{i \in \mathcal{I}} \sum_{j=1}^{N} a_{i j} \tilde{\lambda}_{j i}\left[\rho_{i_{1} \cdots j \cdots i_{n}}-\rho_{i_{1} \cdots i_{n} j}\right]$ 
where $\rho_{i_{1} \cdots j \cdots i_{n}}$ and $\rho_{i_{1} \cdots i_{n} j}$ are the $n$ and $(n+1)$-point correlation functions of the sets $\mathcal{I}_{i} \cup\{j\}$ and $\mathcal{I} \cup\{j\}$, respectively, and where we have also defined

$$
\tilde{\delta}_{i}(t) \equiv\left\langle\delta\left[t_{i}(t)\right] \mid\left\{n_{j}=1, j \in \mathcal{I}\right\}\right\rangle
$$

and

$\tilde{\lambda}_{j i}(t) \equiv\left\langle\lambda\left[\tau_{j i}(t)\right] \mid n_{i}=0, n_{j}=1,\left\{n_{k}=1, k \in \mathcal{I}_{i}\right\}\right\rangle$.

Equations (7)-(9) are the central result of our paper as they fully describe the dynamics of the epidemic. What makes our formulation interesting is the fact that all nonMarkovian effects of the dynamics are encoded in the terms $\tilde{\delta}_{i}$ and $\tilde{\lambda}_{j i}$. As we show later, under certain conditions these parameters take constant values independent of the nodes, that is, $\tilde{\delta}_{i}=\tilde{\delta}$ and $\tilde{\lambda}_{j i}=\tilde{\lambda}$. In this case, the dynamics, even if strongly non-Markovian, can be described by a Markovian one on the same network, using effective parameters $\tilde{\delta}$ and $\tilde{\lambda}$. In this way, the considerable complexity of non-Markovian effects is reduced to the evaluation of such effective parameters.

To proceed further, we need to define the details of the pairwise interaction that rules the infection process. We assume that the infection process between an infected node $j$ and a susceptible node $i$ depends on the state of node $j$ alone; i.e., when a node $j$ becomes infected, it starts an infection process independently to each of his neighbors, regardless of their state, according to a renewal process with interevent time distribution $\psi_{I}(t)$. One can think of this process as a series of firing events, separated by random times $t_{I}$, starting when node $j$ becomes infected, so that when one such event takes place at a time that neighbor node $i$ is susceptible, node $i$ becomes infected (see Fig. 1). We also assume that the recovery process of an infected node depends on its state alone; i.e., when a node becomes infected, it starts a recovery process with random time $t_{R}$, distributed as $\psi_{R}(t)$.

Within this framework, the average of $\lambda\left[\tau_{j i}(t)\right]$ conditioned to the state of the system can be derived by noting that, if $\tau_{j i}$ is the time elapsed since the infection process of node $j$ to node $i$ started and $\tau_{i}^{R}$ is the time elapsed since node $\mathrm{i}$ has recovered, then it may hold that $\tau_{j i}>\tau_{i}^{R}$. Therefore, $\tau_{j i}$ depends on the state of $i$ but not on the state of any other neighbor and, consequently, $\operatorname{Prob}\left(\tau_{j i} ; t \mid n_{i}=0\right.$, $\left.n_{j}=1,\left\{n_{k}=1, k \in \mathcal{I}_{i}\right\}\right)=\operatorname{Prob}\left(\tau_{j i} ; t \mid n_{i}=0, n_{j}=1\right.$ ) (see Supplemental Material [31] for a detailed proof). This implies that we can then define an effective infection rate $\lambda_{\text {eff }}$ as

$$
\begin{aligned}
\lambda_{\text {eff }} & \equiv \lim _{t \rightarrow \infty}\left\langle\lambda\left[\tau_{j i}(t)\right] \mid n_{i}=0, n_{j}=1\right\rangle \\
& =\int_{0}^{\infty} \phi\left(\tau_{j i}\right) \lambda\left(\tau_{j i}\right) d \tau_{j i},
\end{aligned}
$$

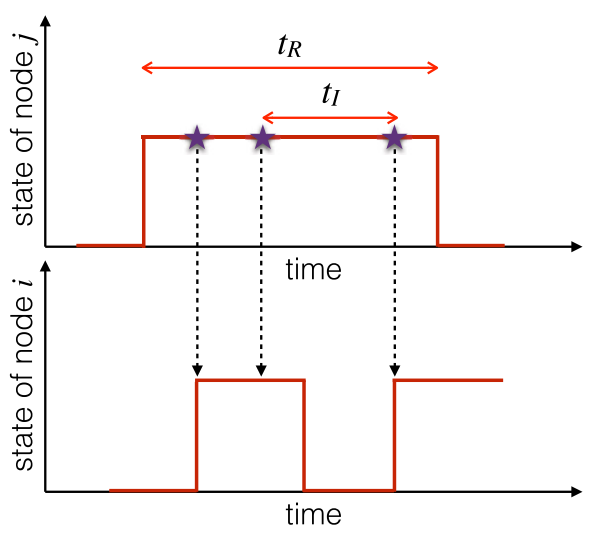

FIG. 1. Sketch of the infection mechanism from node $j$ to node $i$. Infection events triggered by node $j$ (represented by stars in the figure) are ineffective if node $i$ is already infected.

where $\phi\left(\tau_{j i}\right) \equiv \lim _{t \rightarrow \infty} \operatorname{Prob}\left(\tau_{j i} ; t \mid n_{j}=1, n_{i}=0\right)$ is the probability density of $\tau_{j i}$, where $\tau_{j i}$ is the time elapsed since the start of the infection process from node $j$ to node $i$, given that node $i$ is susceptible and node $j$ is infected and $\lambda\left[\tau_{j i}(t)\right]$ is averaged over all active links $i-j$ in the network.

Concerning the average of $\delta\left[t_{i}(t)\right]$, we note that in general, in the long time limit, Eq. (8) does not reduce to Eq. (4), since $\operatorname{Prob}\left(t_{i} ; t \mid\left\{n_{k}=1, k \in \mathcal{I}\right\}\right) \neq \operatorname{Prob}\left(t_{i} ; t \mid n_{i}=1\right)$. This is due to the fact that, especially for low-degree nodes, the time $t_{i}(t)$ may depend on the status of node $i$ 's neighbors: if at time $t$ node $j$ is infected and connected only to node $i\left(k_{j}=1\right)$, node $j$ must have been infected by node $i$; therefore $t_{i}$ has to be larger than the time elapsed since the infection process of node $j$ started. Thus, if the recovery process follows a non-Markovian dynamics it is not possible to define an effective parameter $\delta_{\text {eff }}$. Therefore, hereafter we consider only the case of Markovian recovery, which implies $\tilde{\delta}_{i}=\tilde{\delta}=\left\langle t_{R}\right\rangle^{-1}$, so that the non-Markovian SIS dynamics can be reduced to a Markovian one with parameters $\tilde{\delta}$ and $\lambda_{\text {eff }}$.

Although the probability density $\phi\left(\tau_{j i}\right)$ can be easily measured in numerical simulation, it is too cumbersome to be computed analytically, even in the simplest case of Markovian recovery (see Supplemental Material [31]). Therefore, we also evaluate an approximate effective infection rate $\lambda_{\text {app. }}$. To do so, we first notice that $\lambda_{\text {eff }}$ can also be written as

$$
\lambda_{\text {eff }}=\mathcal{N}^{-1} \int_{0}^{\infty} \psi\left(\tau_{j i}\right) \lambda\left(\tau_{j i}\right) d \tau_{j i},
$$

where $\psi\left(\tau_{j i}\right) \equiv \lim _{t \rightarrow \infty} \operatorname{Prob}\left(\tau_{j i}, n_{i}=0 ; t \mid n_{j}=1\right)$ is the joint probability that node $i$ is susceptible and the time elapsed since the last infection attempt from $j$ to $i$ is equal to $\tau_{j i}$, given that node $j$ is infected at a given observation time $t \rightarrow \infty$, and where $\mathcal{N}$ is the normalization factor 
$\mathcal{N}=\int_{0}^{\infty} \psi\left(\tau_{j i}\right) d \tau_{j i}$. In Supplemental Material [31], we derive an approximate analytic expression for $\psi\left(\tau_{j i}\right)$ that, combined with Eq. (11), allows us to derive the following expression for the approximate effective rate,

$\lambda_{\mathrm{app}}=\frac{\hat{\psi}_{I}(2 \tilde{\delta})+\langle k\rangle \hat{\psi}_{I}(\tilde{\delta})\left[1-\hat{\psi}_{I}(2 \tilde{\delta})\right]\left[\hat{\psi}_{I}(\tilde{\delta})-1\right]^{-1}}{[\langle k\rangle-1] \hat{\Psi}_{I}(\tilde{\delta})}$

where $\hat{\psi}_{I}(u) \equiv \mathcal{L}\left\{\psi_{I}(t)\right\}$ and $\hat{\Psi}_{I}(u) \equiv \mathcal{L}\left\{\Psi_{I}(t)\right\}$ are the Laplace transforms of $\psi_{I}(t)$ and $\Psi_{I}(t)$, respectively, and $\langle k\rangle$ is the average degree of the network substrate.

We check the validity of the effective infection rates, $\lambda_{\text {eff }}$ and $\lambda_{\text {app }}$, by means of extensive numerical simulations of the non-Markovian SIS dynamics; see Supplemental Material [31]. We consider a Poissonian (Markovian) recovery process with rate $\tilde{\delta}$ and an infection process with a Weibull interevent time distribution, that is,

$$
\psi_{I}(t)=\frac{\alpha_{I}}{b}\left(\frac{t}{b}\right)^{\alpha_{I}-1} e^{-(t / b)^{\alpha_{I}}}, \quad \psi_{R}(t)=\tilde{\delta} e^{-\tilde{\delta} t}
$$

with parameter $\alpha_{I}$ controlling the power-law start and tail of the infection interevent time distribution. We choose $b=$ $\left\langle t_{I}\right\rangle\left[\left(\Gamma\left(1+1 / \alpha_{I}\right)\right)\right]^{-1}$, so that $\left\langle t_{I}\right\rangle$ is the average infection time. Hereafter, and without loss of generality, we set the time scale to $\tilde{\delta}=1$. Once the system has reached its steady state, we evaluate $\lambda_{\text {eff }}$ by selecting random time instants along the process. For each time instant, we select all active links, measure the corresponding values of $\tau_{j i}$, and calculate $\lambda_{\text {eff }}$ as the average of the hazard rates $\lambda\left(\tau_{j i}\right)=$ $\left(\alpha_{I} / b\right)\left(\tau_{j i} / b\right)^{\alpha_{I}-1}$. The approximate infection rate $\lambda_{\text {app }}$ is calculated from Eq. (12) by integrating numerically the Laplace transforms $\hat{\psi}_{I}(\tilde{\delta})$ and $\hat{\Psi}_{I}(\tilde{\delta})$ with $\tilde{\delta}=1$.

Figure 2 shows the prevalence $\rho^{\text {st }}$ at the steady state, averaged over all nodes and measured in numerical simulations, as a function of $\lambda_{\text {eff }}$ and $\lambda_{\text {app }}$, for different values of $\alpha_{I}$ and different network substrates: a twodimensional lattice (LATT) with periodic boundary conditions and linear size $L=50$ (so $N=2500$ ), an Erdős Rényi (ER) graph with $\langle k\rangle=8$ and size $N=10^{3}$, a RDR network with $\langle k\rangle=8$ and size $N=10^{3}$, and a scale-free (SF) network with exponent $\gamma=2.5$ and size $N=10^{3}$. One can see that different curves of the prevalence, corresponding to different forms of the infection interevent time distribution, collapse onto one another when plotted as a function of $\lambda_{\text {eff }}$. This result is particularly noteworthy since two infection processes with the same average infection time $\left\langle t_{I}\right\rangle$ but different forms of $\psi_{I}(t)$ are known to behave very differently [17], showing huge differences in the prevalence $\rho^{s t}$ for the same average infection time. This is particularly true in the case of highly heterogeneous processes, such as the one controlled by $\alpha_{I}=0.25$, with a very skewed form of the interevent time distribution $\psi_{I}(t)$,

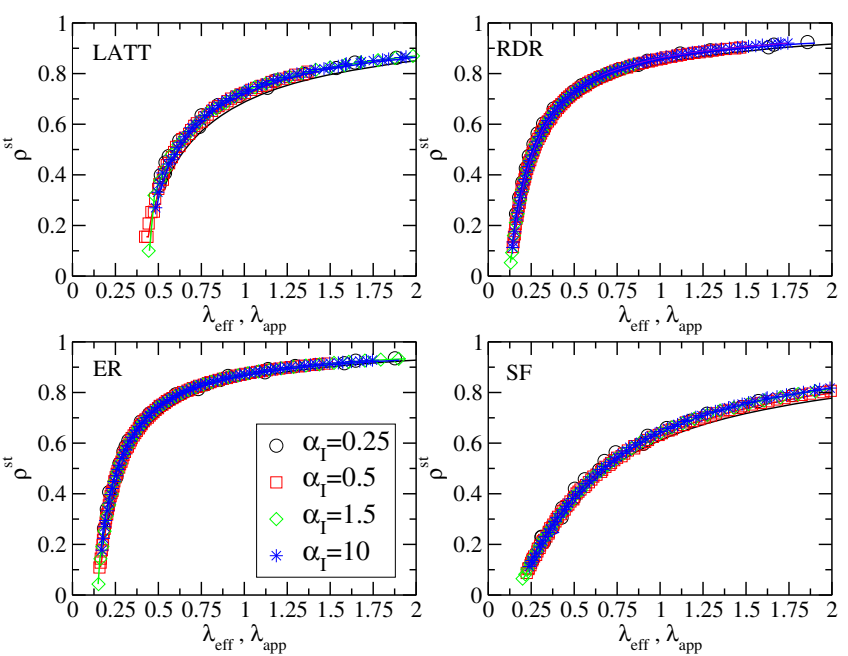

FIG. 2. Steady-state prevalence $\rho^{s t}$ as a function of the effective infection rate, for different values of the exponent $\alpha_{I}$ controlling the interevent time infection distribution and different network substrates. Symbols represent the effective infection rate $\lambda_{\text {eff }}$, extracted by numerical simulations; continuous lines represent the approximate rate $\lambda_{\text {app }}$.

and by $\alpha_{I}=10$, which corresponds to an almost-periodic process.

The curves plotted as functions of the approximate infection rate $\lambda_{\text {app }}$ are also almost indistinguishable from the others, showing that $\lambda_{\text {app }}$ is a very accurate approximation of the exact effective rate, for every underlying network topology. In Supplemental Material [31] we also show that $\lambda_{\text {app }}$ is considerably different from the mean-field approximation proposed in [17], and far more accurate in describing extreme cases such as $\alpha_{I}=0.25$ and $\alpha_{I}=10$; see Supplemental Material [31] Fig. 2. Interestingly, as we show in Supplemental Material [31], Eq. (12) is expected to converge to $\lambda_{\text {eff }}$ in the limit of low prevalence $\rho^{s t} \ll 1$ and, thus, close to the epidemic threshold, $\lambda_{c}$. This implies that the exact critical point $\lambda_{c}$ of the non-Markovian SIS dynamics can be evaluated by means of Eq. (12). Using the same argument, we also conclude that the set of critical exponents of the non-Markovian dynamics is the same as those of the Markovian one.

We check our hypothesis and evaluate the behavior of the non-Markovian SIS dynamics and its critical properties by performing a finite size scaling analysis. We obtain the epidemic threshold $\lambda_{c}$, evaluated by means of Eq. (12), and the set of critical exponents $\beta, \nu_{\perp}$, and $\delta$ for a nonMarkovian SIS dynamics with $\alpha_{I}=0.5$ and $\alpha_{I}=2$, on top of two-dimensional lattice and degree regular networks, by means of the lifespan method proposed in Ref. [32]; see Supplemental Material [31]and Supplemental Material Fig. 3. Table I shows that the critical point and exponents for these cases are in very good agreement with corresponding ones known in the literature for Markovian SIS dynamics. 
TABLE I. Comparison between the critical point $\lambda_{c}$ and critical exponents $\beta, \nu_{\perp}$, and $\delta$ for NM SIS dynamics with different exponent $\alpha_{I}$, and the Markovian case (for which $\alpha_{I}=1$ ), on different underlying network topologies, the two-dimensional lattice and random degree regular (RDR) network. The critical point $\lambda_{c}$ in NM SIS dynamics is evaluated by means of Eq. (12).

\begin{tabular}{lccccl}
\hline \hline Topology & $\alpha_{I}$ & $\lambda_{c}$ & \multicolumn{1}{c}{$\beta$} & \multicolumn{1}{c}{$\nu_{\perp}$} & \multicolumn{1}{c}{$\delta$} \\
\hline Lattice & 0.5 & 0.4194 & 0.596 & 0.739 & 0.453 \\
& 2.0 & 0.4200 & 0.594 & 0.727 & 0.445 \\
& 1.0 & 0.4122 & 0.583 & 0.733 & 0.4505 \\
RDR & 0.5 & 0.3438 & 1.01 & 2.06 & 1.04 \\
& 2.0 & 0.3491 & 1.01 & 2.06 & 1.04 \\
& 1.0 & 0.3452 & 1 & 2 & 1 \\
\hline \hline
\end{tabular}

In conclusion, we have demonstrated that the steady state of non-Markovian SIS dynamics on arbitrary network topologies can be understood in terms of equivalent Markovian dynamics on the same substrates. The possibility of extending our steady-state analysis to transient dynamics remains an open problem. The simplification of the temporal nature of discrete-state processes promises to find application in the wide variety of areas where nonMarkovian aspects are recognized as increasingly influential. It is worth remarking that our formalism is not restricted to the SIS model and can be easily extended to any non-Markovian dynamics with a finite set of discrete states. This allows the determination of the extent to which such dynamics can be reduced to a Markovian equivalent (with redefined parameters) or whether the non-Markovian dynamics are fundamentally different.

We acknowledge support from the James S. McDonnell Foundation; the ICREA Academia prize, funded by the Generalitat de Catalunya; the MINECO Grants No. FIS2013-47282-C2-1-P and No. FIS2016-76830-C22-P; Generalitat de Catalunya Grant No. 2014SGR608; and Science Foundation Ireland Grant No. 11/PI/1026. We thank Xavier Hoffmann for useful discussions.

*Corresponding author.

marian.boguna@ub.edu

${ }^{\dagger}$ Corresponding author.

michele.starnini@gmail.com

[1] F. A. Haight, Handbook of the Poisson Distribution (1967).

[2] A. Barrat, M. Barthélemy, and A. Vespignani, Dynamical Processes on Complex Networks (Cambridge University Press, Cambridge, 2008).

[3] A.-L. Barabasi, Nature (London) 435, 207 (2005).

[4] J. G. Oliveira and A.-L. Barabasi, Nature (London) 437, 1251 (2005).
[5] M. C. Gonzalez, C. A. Hidalgo, and A.-L. Barabasi, Nature (London) 453, 779 (2008).

[6] A. Corral, Phys. Rev. Lett. 92, 108501 (2004).

[7] M. S. Wheatland, P. A. Sturrock, and J. M. McTiernan, Astrophys. J. 509, 448 (1998).

[8] T. Kemuriyama, H. Ohta, Y. Sato, S. Maruyama, M. TandaiHiruma, K. Kato, and Y. Nishida, BioSystems 101, 144 (2010).

[9] D. Bratsun, D. Volfson, L. S. Tsimring, and J. Hasty, Proc. Natl. Acad. Sci. U.S.A. 102, 14593 (2005).

[10] M. Karsai, K. Kaski, A.-L. Barabási, and J. Kertész, Sci. Rep. 2, 397 (2012).

[11] A. Moinet, M. Starnini, and R. Pastor-Satorras, Phys. Rev. Lett. 114, 108701 (2015).

[12] M. Karsai, N. Perra, and A. Vespignani, Sci. Rep. 4, 04001 (2014).

[13] G. García-Pérez, M. Boguñá, and M. Á. Serrano, Sci. Rep. 5, 9714 (2015).

[14] H.-H. Jo, J. I. Perotti, K. Kaski, and J. Kertész, Phys. Rev. X 4, 011041 (2014).

[15] I. Z. Kiss, G. Röst, and Z. Vizi, Phys. Rev. Lett. 115, 078701 (2015).

[16] I. Scholtes, N. Wider, R. Pfitzner, A. Garas, C. J. Tessone, and F. Schweitzer, Nat. Commun. 5, 5024 (2014).

[17] P. Van Mieghem and R. van de Bovenkamp, Phys. Rev. Lett. 110, 108701 (2013).

[18] M. Boguñá, L. F. Lafuerza, R. Toral, and M. A. Serrano, Phys. Rev. E 90, 042108 (2014).

[19] R. Pastor-Satorras, C. Castellano, P. Van Mieghem, and A. Vespignani, Rev. Mod. Phys. 87, 925 (2015).

[20] E. Cator, R. van de Bovenkamp, and P. Van Mieghem, Phys. Rev. E 87, 062816 (2013).

[21] M. Ogura and V. M. Preciado, in 2015 54th IEEE Conference on Decision and Control (CDC) (2015), pp. 3548-3553.

[22] J. L. Iribarren and E. Moro, Phys. Rev. Lett. 103, 038702 (2009).

[23] J. L. Iribarren and E. Moro, Phys. Rev. E 84, 046116 (2011).

[24] K. Lerman, Future Internet 8, 21 (2016).

[25] N. T. J. Bailey, Biometrika 43, 332 (1956).

[26] M. Eichner and K. Dietz, American Journal of Epidemiology 158, 110 (2003).

[27] H. Nishiura and M. Eichner, Epidemiol. Infect. 135, 1145 (2007).

[28] G. Chowell and H. Nishiura, BMC Medicine 12, 1 (2014).

[29] M. Keeling and P. Rohani, Modeling Infectious Diseases in Humans and Animals (Princeton University Press, Princeton, 2007).

[30] D. R. Cox, Renewal Theory (Methuen, London, 1967).

[31] See Supplemental Material at http://link.aps.org/ supplemental/10.1103/PhysRevLett.118.128301 for further information.

[32] M. Boguñá, C. Castellano, and R. Pastor-Satorras, Phys. Rev. Lett. 111, 068701 (2013). 